\title{
Patient privacy: Awareness and attitudes of Iran University of Medical Sciences medical students
}

\author{
Leila Hosseini-Ghavam-Abad ${ }^{1}$, Fariba Asghari ${ }^{2}$, Ali Bandehagh ${ }^{3}$, Sedigheh Najafipour ${ }^{4}$, \\ Shoaleh Bigdeli*5
}

Received: 29 Oct 2017

Published: 4 March 2019

\begin{abstract}
Background: Respecting patients' privacy is an essential professional responsibility for physicians and other health team members. In this regard, this study investigates medical students' knowledge and attitude about confidentiality and disclosure of patients' information.

Methods: In this cross-sectional study, 160 medical students of Iran University of Medical Sciences participated who were selected using stratified random sampling. Data were gathered using a valid and reliable self-report questionnaire. Student's knowledge and attitude toward medical confidentiality were assessed using self-administered and researcher-made questionnaires. Cronbach's alpha coefficients for knowledge and attitude levels were 79.7 and 82.2 , respectively.

Results: The average of medical students' responses to knowledge and attitude questions were $56.6 \%(9.6 / 17)$ and $55.3 \%$ (9.4 out of 17), respectively. On average, females had an acceptable attitude about $57.5 \%$ of the questions, whereas this was $50.9 \%$ for males. On average, females had an acceptable knowledge about $59.5 \%$ of the questions, whereas this was $50.6 \%$ for males. Therefore, female's attitudes and knowledge were more correct than their male counterparts $(\mathrm{p}<0.001)$.

Conclusion: The low level of knowledge and attitude of medical students towards medical confidentiality indicates that revision of Iranian medical education curriculum to reinforce attention and knowledge of medical students on this issue to render appropriate care to patients is a necessity. Medical students' knowledge and attitude towards patient's confidentiality rights is not fulfilling.
\end{abstract}

Keywords: Confidentiality, Knowledge, Attitude

Conflicts of Interest: None declared

Funding: Iran University of Medical Sciences

*This work has been published under CC BY-NC-SA 1.0 license.

Copyright $\odot$ Iran University of Medical Sciences

Cite this article as: Hosseini- Ghavam-Abad L, Asghari F, Bandehagh A, Najafipour S, Bigdeli Sh. Patient privacy: Awareness and attitudes of Iran University of Medical Sciences medical students. Med J Islam Repub Iran. 2019 (4 March);33:12. https://doi.org/10.47176/mjiri.33.12

\section{Introduction}

Privacy and confidentiality are highly emphasized in codes of ethics approved by different medical communities and associations (1); also the World Federation for Medical Education (WFME) has emphasized the necessity to balance academic and behavioral capabilities of medi-

Corresponding author: Dr Shoaleh Bigdeli, bigdeli.sh@iums.ac.ir sbigdeli@alumni.sfu.ca

1. Center for Educational Research in Medical Sciences (CERMS), Iran University of Medical Sciences (IUMS), Tehran, Iran, \& Faculty of Medicine, Iran University of Medical Sciences, Tehran, Iran, \& Department of Health Care Management, Sciences and Research Branch, Islamic Azad University, Semnan, Iran

2. Medical Ethics and History of Medicine Research Center, Tehran University of Medical Sciences, Tehran, Iran

3. Department of Plant Breeding and Biotechnology, Faculty of Agriculture, University of Tabriz, Tabriz, Iran

4. Department of Medical Education, Tehran University of Medical Sciences, Tehran, Iran, \& Medical Educational Center (EDC), Faculty of Medicine, Jahrom University of Medical Sciences, Jahrom, Iran

5. Center for Educational Research in Medical Sciences (CERMS), Iran University of Medical Sciences, Tehran, Iran, \& Faculty of Medicine, Iran University of Medical Sciences, Tehran, Iran cal students (2). Since maintaining the patient's secrets, increases trust and confidence between doctor and patient and promotes the efficiency and effectiveness of medical interventions and treatment processes, to encourage patients to disclose their information properly is important

$\uparrow$ What is "already known" in this topic:

- Confidentiality is fundamental to gain patient's trust.

- Physicians must be adequately aware of the related dilemmas, regulations and codes of confidentiality of their country of practice.

\section{$\rightarrow$ What this article adds:}

- Female interns are more knowledgeable about confidentiality issues than their male counterparts.

- Medical students lack ample knowledge and attitude about patients' confidentiality rights.

- Confidentiality issues must be integrated into the medical curriculum. 
(3). In addition, the Association of American Medical Colleges (AAMC) emphasizes the responsibility of medical schools to train competent students with the ability to make ethical decisions in ethical dilemmas (4). However, commitment to confidentiality is not an absolute responsibility, and in certain circumstances, physicians may be required to ignore the confidentiality of the patient. In order to prevent subjectivity, some countries have specified the situation-relevant cases that can fall under the exception of confidentiality.

In all medical encounters, physicians must ensure patients that their information will remain confidential, and understand that releasing patient's secrets jeopardizes patient trust. The high sensitivity of patient privacy in medical and health professions requires physicians to be adequately aware of the related dilemmas, regulations and confidentiality codes of each country. (1)

In the medical profession, confidentiality is fundamental to gain the patient's trust and help physicians to establish a good rapport with patients. The patient communicates all his/her concerns with the physician, bearing in mind that the physician supports confidentiality and advocates physician-patient relationship. Sometimes, the physician is requested to provide information about the patient's health condition and employability. In these cases, the physician has to keep in mind that $\mathrm{s} / \mathrm{he}$ is not allowed to disclose patient's mental and physical disorders that have been disclosed to her/him or were diagnosed during the examinations without patient's permission. Revealing the patient's secret is a breach of trust and violation of professional responsibility. However, the physician is allowed to compromise her/his responsibilities in individual particular legal cases and under certain terms and conditions. (5)

Keeping patient's secrets confidential is crucial for a trustful doctor-patient relationship that is emphasized by General Medical Council (GMC) and Medical Schools Council, which is patient's right to expect. In many cases, information disclosure is "unplanned and unintentional, and in cases that it must be released in public or social media, anonymity must be assured". Moreover, patient's permission to share his/her information with his/her significant others must be secured before any sharing occurs (6). In this regard, General Medical Council has released a guide entitled "Confidentiality: good practice in handling patient information" on patient privacy and disclosure of information (2017) and expects all doctors to understand and follow it to manage and protect patient information. (7)

The patients' secrets are to remain confidential for a number of reasons, including maintenance of trust and confidence between physician and patient, encouraging patients to refer to a physician or a health center for treatment, and revealing the vital information that facilitates the treatment. Respecting confidentiality increases patient's compliance, respects patient's rights, and prevents social, political and economic discrimination due to patient's health status. This issue, in addition to the role and status of physicians, intensely affect physician-patient relationship. (8) Thus, it is necessary to assess medical students' knowledge and attitude of patients' confidential- ity before they commence practicing medical profession independently. In regard to the importance of the matter, this research is an attempt to discuss the benchmark of knowledge and attitudes of Iranian medical students towards principles of patient's privacy and confidentiality.

\section{Methods}

This analytical cross-sectional study was performed in 2013-2014. The purpose of this study was to determine the level of knowledge and attitude of medical students about confidentiality considerations in patients. Using stratified random sampling, 200 interns of Iran University of Medical Sciences (university entrance years of 2005, 2006 and 2007) were enrolled to participate in the study.

Data collection instruments were a demographic and a self-administered questionnaire developed by Hosseini Ghavamabad et al. (9) which consisted of two sections: (a) Knowledge (17 multiple choice questions), and (b) Attitude (17 statements regarding the same questions of Knowledge section), on a 5 point Likert scale. The questionnaire had face, content and construct validity for both knowledge and attitude sections and confirmatory factor analysis confirms the acceptable validity of all the questions and their appropriateness to collect data.

Ethical approval of the study was obtained from IUMS Ethics Committee. Interns who entered medical school between the years 2005-2007 were identified. The importance of the issue and significance of the application of the research results were explained to them, and qualified students were invited to participate in the research. The students were ensured that their information would remain confidential.

The completed questionnaires were coded, and data analysis was conducted by indices of analysis and testing, and descriptive (percentage frequency distribution, mean and standard error) and inferential statistics (Chi-square, student t-test, Pearson correlation coefficient and Eta correlation ratio). The groups were compared by the Compare Means Test. In all the tests, the confidence coefficient was considered to be 95 percent (level of significance $=0.05$ ). To analyze knowledge, the number of correct answers to the questions of knowledge was calculated as the sum score for each individual. To analyze attitude, the correct direction of attitude in every question was determined. The continuum of strongly agree and agree, and those of strongly disagree and disagree were merged. The questions answered correctly by less than 50 percent of the students were considered as the confidentiality threshold of students' knowledge and attitude weakness. Chi-Square was used to evaluate the effect of gender, marital status, university entrance year and age. To compare mean attitude score by demographic variables, student t-test was used. To illustrate the relationship between quantitative variables, Pearson correlation and for the correlation of qualitative variables, Eta correlation ratio and Chi-Square were used.

\section{Results}

In this study, the response rate was 80 percent (from 200 distributed questionnaires 160 were returned). 
Number of participants who entered the university in 2005, 2006, and 2007 were $45(\% 28.1), 80(\% 50)$ and 35 (\%21.9), respectively. The participants were 108 females $(\% 67.5)$ and 52 males $(\% 32.5)$ with a age range between 23 and 40 years; 129 students were single (\%80.6), and 31 of them were married (\%19.4) (Table 1).

The mean percent of correct responses to the questions of knowledge was $56.6 \%(9.6 / 17)$. The lowest number of correct answers belonged to the question number fifteen which was about revealing the secrets of patients to other physicians without patient's permission (31.9\%). The highest number of correct answers belonged to the question number sixteen $(\% 86.9)$, which was about not revealing the reason for hospitalization of celebrities to other physicians. In sum, the least degree of knowledge and awareness was about revealing patients' information to treating physician, providing information to the patient's trusted physicians, providing information to the police and informing the employer about the medical conditions of the patient. Only 41.2 percent of students knew that the police is not allowed to have full access to the patients' information.

The average percent of correct answers to the questions related to attitude were $55.3 \%$ (9.4 out of 17). The least number of correct answers to attitude was $\% 7.5$, related to the question number 15 , in regard to informing the treating physician about the cause of a sickness which was revealed by the patient to the medical student as a secret. Moreover, the students did not have sound knowledge and appropriate attitude towards not revealing the secrets of patients, especially those affected by AIDS. On average, females had acceptable attitudes about $57.5 \%$ of the questions, whereas this was $50.9 \%$ for males. Therefore, female's attitudes have been more correct than their male counterparts $(\mathrm{p}<0.001)$.

The questions to which the research participants answered incorrectly included: inadequate awareness, providing the journalist with information of diseases of the celebrities and well-known people, revealing the type of disease to the patient family, disclosing the secrets that have been detected during examination by the physician, reporting test results to the police and issuing health certificate in response to requests of employers. Less than 50\% of the participants provided correct answers to the mentioned questions, which is indicative of inadequate knowledge. Moreover, regarding the attitude section, there was an inappropriate attitude toward matters such as keeping the patients' information from other researchers as a secret, not disclosing the patient's sickness to her/his relatives and friends, and keeping the secrets that are diagnosed during the patient examination.

\section{Discussion}

This research has been carried out to determine the degree of knowledge and attitude of interns regarding patient confidentiality. In a similar research done on physicians, nurses and faculty members of McGill University, the most important finding was lack of staff awareness about police easy access to information of patients. (10)

From the point of view of the study participants, if a patient secretly talks about his/her cause of illness, it is the student responsibility to explain it to the treating physician regardless of the patient's permission $(69.1 \%$ of the students incorrectly answered this question). In the same vein, in the attitude section, only $\% 7.5$ of the study participants had chosen the correct answer. As a result, different levels of attitude and knowledge were observed among students, and they unconsciously ignored confidentiality rules. Also, the students believed that the patient's family and friends are his/her confidant; hence, physicians are allowed to inform them about the health status of the patient, e.g. HIV test.

In Ian Shrier's research, a high percentage of the participants had a low degree of familiarity with confidentiality rules, and in cases where there was a contradiction between knowledge and attitude prediction of the subsequent behavior was a hurdle. (10) In another research on patient confidentiality, Moridzadeh found that half of the general practitioners, scored less than $\% 50$ of total and the mean of their scores was 51 percent. (11) The present study showed that female interns were more knowledgeable than their male counterparts which is similar to Moridzadeh's research findings.

Another research in teaching hospitals of Khorramabad (Iran) on the familiarity of physicians and nurses to legal aspects of patients' privacy indicates that the participants' overall awareness of correct responses was $42.1 \%$; and $7.5 \%$ of them did not answer the questions related to the legal aspects of confidentiality. Concerning disclosure of patient's secrets, the highest degree of unawareness was $65.6 \%$, which indicates low awareness of the participants. However, these differences may be due to the adminis-

Table 1. The knowledge and attitude of medical students toward confidentiality according to their demographic characteristics

\begin{tabular}{|c|c|c|c|c|c|c|}
\hline Demographic variables & $\begin{array}{l}\text { Variable } \\
\text { classes }\end{array}$ & Numbers & $\begin{array}{l}\text { The average percent of correct } \\
\text { answers to questions of } \\
\text { knowledge }\end{array}$ & $\mathrm{p}$ & $\begin{array}{l}\text { The average percent of correct } \\
\text { answers to questions of attitude }\end{array}$ & $\bar{p}$ \\
\hline \multirow[t]{2}{*}{ Gender } & Female & 108 & $59.5 \%$ & $<0.001$ & $57.5 \%$ & 0.001 \\
\hline & Male & 52 & $50.6 \%$ & & $50.9 \%$ & \\
\hline \multirow[t]{2}{*}{ Marital Status } & Single & 129 & $56.7 \%$ & 0.816 & $54.8 \%$ & 0.226 \\
\hline & Married & 31 & $56.2 \%$ & & $57.7 \%$ & \\
\hline \multirow[t]{3}{*}{ Age } & $20,25,26,30$ & 82 & $57.7 \%$ & 0.475 & $58.8 \%$ & 0.181 \\
\hline & and $>30$ & 76 & $55.4 \%$ & & $51.7 \%$ & \\
\hline & & 2 & $55.9 \%$ & & $55.9 \%$ & \\
\hline University entrance & 84 & 45 & $60.1 \%$ & 0.049 & $54.1 \%$ & 0.488 \\
\hline \multirow{2}{*}{ Year } & 85 & 80 & $54.6 \%$ & & $56.5 \%$ & \\
\hline & 86 & 35 & $56.6 \%$ & & $54.3 \%$ & \\
\hline
\end{tabular}


tered research instruments. (12) Sheikhtaheri et al. study on physicians' awareness of legal aspects of patients' privacy in teaching hospitals of Kashan (Iran) in 2007 indicated that only $37.3 \%$ of the physicians had provided correct answers to patient's privacy questions. (13) In another research on hospitalized patients, the percentage of respecting patient's rights on privacy of medical information was 63.05 percent. (14)

In another study, culture, religion, and point of views of inhabitants of a region were considered as the basis of modern medical practice. (15) Moreover, Sanker et al. in a study on adolescents and vulnerable groups (e.g. mentally ill, HIV positive, battered women, etc.) found that these groups were reluctant to report their illness due to privacy and confidentiality concerns. (16)

Ian Shrier's research results indicated that despite patient satisfaction of the role of physicians in their treatment, they prefer their information to remain confidential. The results of this research yield that there is a low degree of awareness in university systems about confidentiality. (10) Yousuf et al. in a cross-sectional comparative study on physicians' awareness, knowledge and attitude towards securing informed consent, found that all respondents believed that although it is less probable that patients reveal all their secrets, their diagnosis should not be released.

\section{Conclusion}

The findings of this research indicated that there are some deficiencies in medical students' knowledge and attitude in regard to patients' confidentiality rights. Therefore, enrichment and development of the medical curriculum will promote the educational attainment of medical students in relation to confidentiality and privacy of patients, Iran Ministry of Health and Medical Education that is responsible for centralized medical curriculum revisions should consider the importance of confidentiality of patients' information in the curriculum to educate knowledgeable physicians with positive attitude about enacting ethical rules in their practice.

Moreover, in regard to the special features of medical ethics and its crucial importance, the medical curriculum must include ethical codes and guide professional behavior of physicians; therefore, the issue of confidentiality must be integrated into medical curriculums.

\section{Study Limitation}

The study was conducted in one medical school. It is suggested that the questionnaire to be distributed in other medical universities of the country to gain more data in this regard. In addition, the sampling method was not random, and this might reduce the generalizability of the findings.

\section{Acknowledgments}

We would like to thank the Center for Educational Research in Medical Education (CERMS) affiliated to Iran University of Medical Sciences for its financial support to implement this project (Reg. No. 24155). We are also thankful to all the study participants. We sincerely express our gratitude to Ms. Afandideh (IUMS medical school education staff) and Ms. Sabet Rouhani (head staff of the department of community medicine, school of medicine, IUMS), and Dr. Raf'ati, Rasul Akram Hospital manager for their sincere support.

\section{Conflict of Interests}

The authors declare that they have no competing interests.

\section{References}

1. Parsa M. Privacy and confidentiality in medical field and its various aspects. J Med Ethics Hist. 2009;4:1-14.

2. Hosseini GH, Asghari F, BandehHagh A, Bigdeli Sh, Najafpour S. Designing a Valid and Reliable Tool to Assess Knowledge and Attitudes of Medical Students [MSc dissertation]. Iran University of Medical Sciences; 2014.

3. Siavosh B. A comparative study on the access levels and confidentiality of medical records among selected countries and Iran [MSc dissertation] Iran University of Medical Sciences; 2005.

4. Khajedaluee M, Movafaghi Z, Pouryazdanpanah M. Effectiveness of the medical ethics curriculum: viewpoints of interns in Mashhad University of Medical science. Mec Etics His Med. 2013;6(5):67-76.

5. Ashrafi MN, Ashrafi MR. Medical ethics and professional misconduct. 1st Ed. Higher Institute of Educational Research and Planning Organization Publishers; 2005: pp.137-163.

6. General Medical Council. Achieving Good Medical Practice: Guidance for Medical Students. GMC/MSC; 2016.

7. Confidentiality: good practice in handling patient information [homepage on the Internet]. Available from: https://www.gmcuk.org/ethical-guidance/ethical-guidance-for-doctors/confi

8. Larijani B, Jafarian A, Kazemian A, Sadr hoseini M. Medical and ethical considerations. 2nd Ed. Publications for tomorrow. 2004. pp. $73-80$

9. Bigdeli S, Hosseini-Qavam-Abad L, Asghari F, Bandehagh A, Najafipour S. Designing a Tool to Assess Medical Students' Knowledge and Attitudes of Confidentiality and Disclosure of Patient Information. J Med Edu. 2016 Jan 1;15(2):75-80.

10. Shrier I, Green S, Solin J, Duarte-Franco E, Guibert R, Brousseau G, Khanlou N. Knowledge of and attitude toward patient confidentiality within three family medicine teaching units. Acad Med. 1998 Jun;73(6):710-2.

11. Moridzadeh P. The Knowledge of physicians participating in educational congresses held in the conference hall of the mystery of the medical confidentiality and disclosure of secrets [MD dissertation]. Iran University of Medical Sciences, 2003.

12. Razavi N, Bahshaee MR. Knowledge of Medical group and Nurses about Patient Bill of Rights in Ekbatan Hospital 1999, Proceedings of the National Conference on the management of health services, 2000.

13. Sheikh Taheri A, Kymyafr Kh, Barati Marnani A. Knowledge of physicians, nurses and staff of medical records of the legal aspects of medical records in hospitals affiliated to Tehran University of Medical Sciences. Health Inform Manag. 2010;7(2):146-136.

14. Rangrazr F, Rabie R. Evaluation of patients' rights in public hospitals in Kashan in 2002. J Kermanshah Uni Med Sci. 2005;1(9):62-71.

15. Yousuf RM, Fauzi AR, How SH, Rasool AG, Rehana K. Awareness, knowledge and attitude towards informed consent among doctors in two different cultures in Asia: a cross-sectional comparative study in Malaysia and Kashmir, India. Singapore Med J. 2007 Jun;48(6):559.

16. Sankar P, Mora S, Merz JF, Jones NL. Patient perspectives of medical confidentiality: a review of the literature. J Gen Intern Med. 2003 Aug;18(8):659-69. 